(1)

\title{
Nonclassical Symmetries for a Class of Reaction-Diffusion Equations: the Method of Heir-Equations
}

M.S. Hashemi, M.C. Nucci

To cite this article: M.S. Hashemi, M.C. Nucci (2013) Nonclassical Symmetries for a Class of Reaction-Diffusion Equations: the Method of Heir-Equations, Journal of Nonlinear Mathematical Physics 20:1, 44-60, DOI:

https://doi.org/10.1080/14029251.2013.792469

To link to this article: https://doi.org/10.1080/14029251.2013.792469

Published online: 04 January 2021 


\title{
Nonclassical Symmetries for a Class of Reaction-Diffusion Equations: the Method of Heir-Equations
}

\author{
M.S. Hashemi \\ Department of Mathematics \\ Imam Khomeini International University \\ Ghazvin 34149, Iran \\ hashemi_math396@yahoo.com \\ M.C. Nucci \\ Dipartimento di Matematica e Informatica \\ Università degli Studi di Perugia \& INFN Sezione di Perugia \\ 06123 Perugia, Italy \\ nucci@unipg.it \\ Received 4 September 2012 \\ Accepted 5 November 2012
}

\begin{abstract}
The nonclassical symmetries method is applied to a class of reaction-diffusion equations with nonlinear source, i.e. $u_{t}=u_{x x}+c u_{x}+R(u, x)$. Several cases are obtained by using suitable solutions of the heir-equations as described in [M.C. Nucci, Nonclassical symmetries as special solutions of heir-equations, J. Math. Anal. Appl. 279 (2003) 168-179].
\end{abstract}

Keywords: reaction-diffusion equations; Nonclassical symmetries; heir-equations

2010 Mathematics Subject Classification: 35K57, 58D19, 35C05

\section{Introduction}

In a recent paper [5] a class of reaction-diffusion equations, i.e.

$$
u_{t}=u_{x x}+c u_{x}+R(u, x),
$$

with $R(u, x)$ arbitrary function of $u$ and $x$, was introduced as a model that incorporates climate shift, population dynamics, and migration for a population of individuals $u(t, x)$ that reproduce, disperse, and die within a patch of favorable habitat surrounded by unfavorable habitat. It is assumed that due to a shifting climate, the patch moves with a fixed speed $c>0$ in a one-dimensional universe ${ }^{\mathrm{a}}$.

Motivated by this study here we look for nonclassical symmetries of equation (1.1) with the purpose of finding explicit expressions of the function $R(u, x)$ and deriving nonclassical symmetry solutions when feasible.

Nonclassical symmetries were introduced in 1969 in a seminal paper by Bluman and Cole [8]. After twenty years and few occasional papers, e.g. [50], [9], in the early Nineties there was a sudden spur of interest and several papers began to appear, e.g. [34], [20], [41], [40], [48], [52], [36], [26],

$\overline{{ }^{a} \text { Actually equation (1.1) }}$ corresponds to the original model

$$
u_{t}=u_{z z}+R(u, z-c t)
$$

rewritten in terms of a moving coordinate system with $x=z-c t$ [5]. 
[18], [19], [2], [47], [3], [24], [22]. Since then the nonclassical symmetry method has been applied to various equations and systems in hundreds of published papers, e.g. [27], [37], [16], [28], [17], [54], [13], [11], [12], [15], [51], [4], the latest ${ }^{\mathrm{b}}$ being [14], [31], [55], [10].

One should be aware that some authors call nonclassical symmetries as $Q$-conditional symmetries $^{\mathfrak{c}}$ of the second type, e.g. [13], while others call them reduction operators, e.g. [51].

The nonclassical symmetry method can be viewed as a particular instance of the more general differential constraint method that, as stated by Kruglikov [33], dates back at least to the time of Lagrange... and was introduced into practice by Yanenko [57]. The method was set forth in details in Yanenko's monograph [53] that was not published until after his death [21]. A more recent account and generalization of Yanenko's work can be found in [39].

Among the papers dedicated to the application of the nonclassical symmetry method to diffusion-convection equation with source, we single out [11] where some nonclassical symmetries solutions were determined for the equation:

$$
u_{t}=u_{x x}+k(x) u^{2}(1-u) .
$$

In particular nonclassical symmetries of the type $V(t, x) \partial_{x}+\partial_{t}$ were found in the following three instances:

$$
\begin{array}{lll}
\text { (i) } k(x)=a^{2} x^{2}, & \text { (ii) } k(x)=a^{2} \tanh ^{2} x, & \text { (iii) } k(x)=a^{2} \tan ^{2} x
\end{array}
$$

with $a$ arbitrary constant.

In the next Section we recall the concept of heir-equations [43] and their link to nonclassical symmetries [46]. In Section 3 the nonclassical symmetries of equation (1.1) are reported, along with the corresponding reductions and solutions. The last Section contains some final remarks.

\section{Heir-equations and nonclassical symmetries}

Let us consider an evolution equation in two independent variables and one dependent variable of second order:

$$
u_{t}=H\left(t, x, u, u_{x}, u_{x x}\right)
$$

If

$$
\Gamma=V_{1}(t, x, u) \partial_{t}+V_{2}(t, x, u) \partial_{x}-F(t, x, u) \partial_{u}
$$

is a generator of a Lie point symmetry ${ }^{\mathrm{d}}$ of equation (2.1) then the invariant surface condition is given by:

$$
V_{1}(t, x, u) u_{t}+V_{2}(t, x, u) u_{x}=F(t, x, u) .
$$

\footnotetext{
${ }^{b}$ Namely papers published within the first half of 2012 .

${ }^{c}$ In [25] this name was introduced for the first time.

${ }^{\mathrm{d}}$ The minus sign in front of $F(t, x, u)$ was put there for the sake of simplicity: it could be replaced with a plus sign without affecting the following results.
} 
Let us take the case with $V_{1}=0$ and $V_{2}=1$, so that (2.3) becomes ${ }^{\mathrm{e}}$ :

$$
u_{x}=G(t, x, u)
$$

Then, an equation for $G$ is easily obtained. We call this equation $G$-equation [42]. Its invariant surface condition is given by:

$$
\xi_{1}(t, x, u, G) G_{t}+\xi_{2}(t, x, u, G) G_{x}+\xi_{3}(t, x, u, G) G_{u}=\eta(t, x, u, G)
$$

Let us consider the case $\xi_{1}=0, \xi_{2}=1$, and $\xi_{3}=G$, so that (2.5) becomes:

$$
G_{x}+G G_{u}=\eta(t, x, u, G)
$$

Then, an equation for $\eta$ is derived. We call this equation $\eta$-equation. Clearly:

$$
G_{x}+G G_{u} \equiv u_{x x} \equiv \eta
$$

We could keep iterating to obtain the $\Omega$-equation, which corresponds to:

$$
\eta_{x}+G \eta_{u}+\eta \eta_{G} \equiv u_{x x x} \equiv \Omega(t, x, u, G, \eta)
$$

the $\rho$-equation, which corresponds to:

$$
\Omega_{x}+G \Omega_{u}+\eta \Omega_{G}+\Omega \Omega_{\eta} \equiv u_{x x x x} \equiv \rho(t, x, u, G, \eta, \Omega)
$$

and so on. Each of these equations inherits the symmetry algebra of the original equation, with the right prolongation: first prolongation for the $G$-equation, second prolongation for the $\eta$-equation, and so on. Therefore, these equations were named heir-equations in [43]. This implies that even in the case of few Lie point symmetries many more Lie symmetry reductions can be performed by using the invariant symmetry solution of any of the possible heir-equations, as it was shown in [43], [1], [38].

We recall that the heir-equations are just some of the many possible $n$-extended equations as defined by Guthrie in [30].

In [43] it was shown that this iterating method yields both partial symmetries as given by Vorobev in [56], and differential constraints as given by Olver [49].

Fokas and Liu [23] and Zhdanov [58] independently introduced the method of generalised conditional symmetries, i.e., conditional Lie-Bäcklund symmetries. In [44] it was shown that the heirequations can retrieve all the conditional Lie-Bäcklund symmetries found by Zhdanov.

In [29] Goard has shown that Nucci's method of constructing heir equations by iterating the nonclassical symmetries method is equivalent to the generalised conditional symmetries method.

The difficulty in applying the method of nonclassical symmetries consists in solving nonlinear determining equations in contrast with the linearity of the determining equations in the case of classical symmetries.

The concept of Gröbner basis has been used [19] for this purpose.

In [46] it was shown that one can find the nonclassical symmetries of any evolution equations of any order by using a suitable heir-equation and searching for a given particular solution among all its solutions, thus avoiding any complicated calculations. We recall the method as applicable to equation (2.1).

${ }^{\mathrm{e}}$ We have replaced $F(t, x, u)$ with $G(t, x, u)$ in order to avoid any ambiguity in the following discussion. 
We derive $u_{t}$ from (2.1) and replace it into (2.3), with the condition $V_{1}=1$, i.e.:

$$
H\left(t, x, u, u_{x}, u_{x x}\right)+V_{2}(t, x, u) u_{x}=F(t, x, u)
$$

Then, we generate the $\eta$-equation with $\eta=\eta(x, t, u, G)$, and replace $u_{x}=G, u_{x x}=\eta$ into (2.10), i.e.:

$$
H(t, x, u, G, \eta)=F(t, x, u)-V_{2}(t, x, u) G
$$

For Dini's theorem, we can isolate $\eta$ in (2.11), e.g.:

$$
\eta=\left[h_{1}(t, x, u, G)+F(t, x, u)-V_{2}(t, x, u) G\right] h_{2}(t, x, u, G)
$$

where $h_{i}(t, x, u, G)(i=1,2)$ are known functions. Thus, we have obtained a particular solution of $\eta$ which must yield an identity if replaced into the $\eta$-equation. The only unknowns are $V_{2}=V_{2}(t, x, u)$ and $F=F(t, x, u)$. If any such solution is singular, i.e. does not form a group then we have found the nonclassical symmetries, otherwise one obtains the classical symmetries [46].

More recently in [7] Bîlă and Niesen presented another method that reduces the partial differential equation (PDE) to an ordinary differential equation by using the invariant surface condition and then applies the Lie classical symmetry method in order to find nonclassical symmetries of the original PDE. We hope that an independent researcher will take up the task of comparing the two methods as it was done by Goard in [29] since we conjecture that Bîlă and Niesen's method, and its extension, as given in [12], are equivalent to Nucci's method [46].

\section{Nonclassical symmetries of (1.1)}

We use a simple MAPLE program to derive the heir-equations. In particular the $G$-equation of (1.1) is:

$$
G_{t}+R G_{u}-G_{x x}-2 G G_{x u}-G^{2} G_{u u}-c G_{x}-R_{u} G-R_{x}=0 .
$$

and the $\eta$-equation is

$$
\begin{gathered}
\eta_{t}+R \eta_{u}+R_{u} G \eta_{G}-\eta_{x x}-2 G \eta_{x u}-2 \eta \eta_{x G}-G^{2} \eta_{u u}-2 G \eta \eta_{u G} \\
-\eta^{2} \eta_{G G}-c \eta_{x}-R_{u u} G^{2}-R_{u} \eta-2 G R_{x u}+R_{x} \eta_{G}-R_{x x}=0 .
\end{gathered}
$$

The particular solution of the $\eta$-equation that we are looking for is

$$
\eta(t, x, u, G)=-R(u, x)-c G+F(t, x, u)-V_{2}(t, x, u) G,
$$

that replaced into (3.2) yields an overdetermined system in the unknowns $F, V_{2}$ and $R(u, x)$. Since we obtain a polynomial of third degree in $G$ then we let MAPLE evaluate the four coefficients that we call $d_{i}, i=0,1,2,3$ where $i$ stands for the corresponding power of $u$. We impose all of them to 
be zero. From $d_{3}$, we obtain

$$
V_{2}(t, x, u)=s s_{1}(t, x) u+s s_{2}(t, x)
$$

while $d_{2}$ yields

$$
F(t, x, u)=-\frac{1}{3} s s_{1}^{2} u^{3}+\frac{1}{2}\left(\frac{\partial s s_{1}}{\partial x}-2 c s s_{1}-2 s s_{1} s s_{2}\right)+s s_{3}(t, x) u+s s_{4}(t, x),
$$

with $s s_{j}(t, x), \quad j=1, \ldots, 4$ arbitrary functions of $t$ and $x$. Then after differentiating $d[1]$ four times with respect to $u$ we obtain

$$
\frac{\partial^{4} R(u, x)}{\partial u^{4}}=0
$$

which implies that $R(u, x)$ must be a polynomial in $u$ of third degree at most, i.e.

$$
R(u, x)=-\frac{a_{3}^{2}(x)}{6} u^{3}+\frac{a_{2}(x)}{2} u^{2}+a_{1}(x) u+a_{0}(x),
$$

where $a_{i}(x), \quad i=0,1,2,3$ are arbitrary functions of $x$. Since none of the remaining arbitrary functions depends on $u$, and $d_{1}$ has now become a polynomial of degree 3 in $u$, we have to annihilate all the four coefficients, i.e. $d_{1, i}, \quad i=0,1,2,3$. From $d_{1,3}$ we have that $s s_{1}(t, x)$ must be a constant, and two cases raise:

Case 1. $s s_{1}= \pm \frac{\sqrt{3}}{2} a_{3}(x)$,

Case 2. $s s_{1}=0$.

We discuss the two cases ${ }^{\mathrm{f}}$, separately. We remark that $a_{3}(x)=0$ corresponds to a subcase of Case 2., and consequently in Case 1 . we assume $a_{3}(x) \neq 0$.

Interestingly enough in Case 2. nonclassical symmetries exist for

$$
R(u, x)=\frac{f(u)}{k^{2}(x)}
$$

with $f(u)$ any arbitrary function of $u$, and $k(x)$ either of the following three particular functions of $x$, i.e.

$$
k(x)=-\frac{c x+2}{2 x}, \quad k(x)=\frac{c}{e^{c\left(b_{0}-x\right)}-1}, \quad k(x)=\frac{1}{b_{1}} \tan \left(\frac{x+b_{2}}{b_{1}}\right)-\frac{c}{2}
$$

$\overline{\mathrm{f}}$ In Case 1., one can choose either the plus or minus sign indifferently. 
3.1. Case 1. $R(u, x)=-\frac{a_{3}^{2}(x)}{6} u^{3}+\frac{a_{2}(x)}{2} u^{2}+a_{1}(x) u+a_{0}(x)$

From coefficients $d_{1,2}, d_{1,1}, d_{1,0}$ we obtain $s s_{2}, s s_{3}$, and $s s_{4}$, respectively. All of them are function of $x$ only, e.g.

$$
s s_{2}=-\frac{1}{2 a_{3}(x)}\left(-4 a_{3}^{\prime}(x)+\sqrt{3} a_{2}(x)+2 c a_{3}(x)\right),
$$

where ' denotes differentiation with respect to $x$. Now the only remaining coefficient is $d_{0}$ which has become a linear polynomial in $u$. Therefore we are left with two expressions to annihilate, namely the following underdetermined system of two equations that contain the derivative of $a_{3}(x)$ up to fifth order, and fourth order, respectively, and lower derivatives of the other three functions $a_{2}(x)$, $a_{1}(x)$, and $a_{0}(x)$

$$
\begin{aligned}
& -a_{3}^{3} a_{3}^{(i v)}-4 a_{3}^{3} a_{3}^{\prime \prime} a_{1}-c^{2} a_{3}^{3} a_{3}^{\prime \prime}-5 a_{2}^{2} a_{3} a_{3}^{\prime \prime}+3 a_{2} a_{3}^{2} a_{2}^{\prime \prime}+a_{3}^{3} \sqrt{3} a_{2}^{\prime \prime \prime}-36 a_{3}\left(a_{3}^{\prime}\right)^{2} a_{3}^{\prime \prime} \\
& -2 a_{3}^{3} a_{3}^{\prime \prime \prime} c+8 a_{3}^{2} a_{3}^{\prime} a_{3}^{\prime \prime \prime}+a_{0}^{\prime} \sqrt{3} a_{3}^{5}-2 a_{3}^{\prime} a_{3}^{3} a_{1}^{\prime}-18 a_{3}^{\prime 3} \sqrt{3} a_{2}+c a_{3}^{4} a_{1}^{\prime}+4 a_{3}^{2} a_{3}^{\prime 2} a_{1} \\
& -a_{2}^{3} \sqrt{3} a_{3}^{\prime}-2 a_{2} \sqrt{3} a_{3}^{2} a_{3}^{\prime \prime} c+16 a_{3} a_{3}^{\prime} a_{3}^{\prime \prime} \sqrt{3} a_{2}+14 a_{3}^{2} a_{3}^{\prime} a_{3}^{\prime \prime} c+3 a_{3}^{2} a_{2}^{\prime 2}+5 a_{3}^{2} a_{3}^{\prime \prime 2} \\
& +c a_{3}^{3} \sqrt{3} a_{2}^{\prime \prime}-5 a_{3}^{2} a_{3}^{\prime} \sqrt{3} a_{2}^{\prime \prime}-6 a_{3}^{2} a_{3}^{\prime \prime} \sqrt{3} a_{2}^{\prime}-2 a_{2} \sqrt{3} a_{3}^{2} a_{3}^{\prime \prime \prime}+13 a_{3}^{\prime 2} a_{2}^{2}+a_{1}^{\prime} a_{3}^{3} \sqrt{3} a_{2} \\
& +a_{3}^{3} \sqrt{3} a_{2}^{\prime} a_{1}+14 a_{3} a_{3}^{\prime 2} \sqrt{3} a_{2}^{\prime}-a_{2}^{2} a_{3} c a_{3}^{\prime}+a_{0} \sqrt{3} a_{3}^{4} a_{3}^{\prime}+a_{2}^{2} \sqrt{3} a_{3} a_{2}^{\prime} \\
& -12 a_{3} a_{3}^{\prime 3} c+c^{2} a_{3}^{2} a_{3}^{\prime 2}+4 a_{3} a_{3}^{\prime 2} c \sqrt{3} a_{2}-a_{3}^{2} a_{3}^{\prime} \sqrt{3} a_{2} a_{1}-3 a_{3}^{2} c a_{3}^{\prime} \sqrt{3} a_{2}^{\prime} \\
& +24 a_{3}^{\prime 4}-14 a_{3} a_{3}^{\prime} a_{2} a_{2}^{\prime}+a_{2} a_{3}^{2} c a_{2}^{\prime}+3 a_{3}^{4} a_{1}^{\prime \prime}=0, \\
& \frac{6}{\sqrt{3}} a_{3}^{4} a_{3}^{(v)}+7 c \sqrt{3} a_{3}^{2} a_{3}^{\prime} a_{2}^{\prime} a_{2}-c a_{3}^{6} a_{0}^{\prime}-3 a_{3}^{\prime 2} a_{2}^{3}+192 \sqrt{3} a_{3}^{\prime 5}-26 c \sqrt{3} a_{3}^{3} a_{3}^{\prime \prime \prime} a_{3}^{\prime} \\
& +2 c \sqrt{3} a_{3}^{4} a_{1}^{\prime} a_{3}^{\prime}-264 a_{3}^{\prime 4} a_{2}+5 a_{3}^{3} a_{3}^{(i v)} a_{2}+104 \sqrt{3} a_{3}^{\prime 2} a_{3}^{2} a_{3}^{\prime \prime \prime}-18 \sqrt{3} a_{3}^{3} a_{3}^{(i v)} a_{3}^{\prime} \\
& +2 c^{2} \sqrt{3} a_{3}^{4} a_{3}^{\prime \prime \prime}+4 c \sqrt{3} a_{3}^{4} a_{3}^{(i v)}-2 c \sqrt{3} a_{3}^{5} a_{1}^{\prime \prime}-26 c \sqrt{3} a_{3}^{3} a_{3}^{\prime \prime 2}+19 c a_{3}^{3} a_{2}^{\prime \prime} a_{3}^{\prime}+27 c a_{3}^{3} a_{2}^{\prime} a_{3}^{\prime \prime} \\
& +6 c a_{3}^{3} a_{3}^{\prime \prime \prime} a_{2}+c^{2} a_{3}^{3} a_{3}^{\prime \prime} a_{2}-416 \sqrt{3} a_{3}^{\prime 3} a_{3} a_{3}^{\prime \prime}+10 \sqrt{3} a_{3}^{4} a_{3}^{\prime \prime} a_{1}^{\prime}+178 \sqrt{3} a_{3}^{2} a_{3}^{\prime \prime 2} a_{3}^{\prime} \\
& -42 \sqrt{3} a_{3}^{3} a_{3}^{\prime \prime} a_{3}^{\prime \prime \prime}-6 a_{3}^{3} \sqrt{3} a_{2}^{\prime} a_{2}^{\prime \prime}+\sqrt{3} a_{3}^{2} a_{3}^{\prime \prime \prime} a_{2}^{2}-\sqrt{3} a_{3}^{3} a_{2}^{\prime \prime \prime} a_{2}+350 a_{3}^{\prime 2} a_{3} a_{3}^{\prime \prime} a_{2}-204 a_{3}^{2} a_{3}^{\prime \prime} a_{2}^{\prime} a_{3}^{\prime} \\
& -56 a_{3}^{\prime} a_{2} a_{3}^{2} a_{3}^{\prime \prime \prime}+2 a_{1} \sqrt{3} a_{3}^{4} a_{3}^{\prime \prime \prime}+5 a_{1} a_{3}^{3} a_{3}^{\prime \prime} a_{2}-54 c a_{3}^{2} a_{3}^{\prime} a_{3}^{\prime \prime} a_{2}-c^{2} a_{3}^{4} a_{2}^{\prime \prime}-4 c a_{3}^{4} a_{2}^{\prime \prime \prime} \\
& -2 \sqrt{3} a_{3}^{5} a_{1}^{\prime \prime \prime}+3 a_{0} a_{3}^{5} a_{3}^{\prime \prime}-88 a_{3}^{\prime 2} a_{3}^{2} a_{2}^{\prime \prime}+39 a_{3}^{3} a_{3}^{\prime \prime} a_{2}^{\prime \prime}-55 a_{3}^{2} a_{3}^{\prime \prime 2} a_{2}+24 a_{3}^{3} a_{2}^{\prime} a_{3}^{\prime \prime \prime} \\
& +20 a_{3}^{3} a_{2}^{\prime \prime \prime} a_{3}^{\prime}-3 a_{1} a_{3}^{4} a_{2}^{\prime \prime}-4 a_{0}^{\prime} a_{3}^{5} a_{3}^{\prime}+34 \sqrt{3} a_{3}^{\prime 3} a_{2}^{2}-4 a_{3}^{\prime 2} a_{3}^{4} a_{0}+232 a_{3}^{\prime 3} a_{3} a_{2}^{\prime} \\
& -6 a_{3}^{4} a_{2}^{\prime} a_{1}^{\prime}-3 a_{3}^{2} a_{2}^{\prime 2} a_{2}-a_{1} a_{3}^{4} c a_{2}^{\prime}+6 c^{2} \sqrt{3} a_{3}^{2} a_{3}^{\prime 3}-2 c \sqrt{3} a_{3}^{3} a_{2}^{\prime 2}-64 c \sqrt{3} a_{3} a_{3}^{\prime 4} \\
& +3 c^{2} a_{3}^{3} a_{3}^{\prime} a_{2}^{\prime}-3 c^{2} a_{3}^{2} a_{3}^{\prime 2} a_{2}-56 c a_{3}^{2} a_{3}^{\prime 2} a_{2}^{\prime}+62 c a_{3} a_{3}^{\prime 3} a_{2}-12 \sqrt{3} a_{3}^{\prime 2} a_{3}^{3} a_{1}^{\prime}+16 a_{3}^{2} \sqrt{3} a_{2}^{\prime 2} a_{3}^{\prime} \\
& -a_{0} a_{3}^{5} a_{3}^{\prime} c+a_{0}^{\prime} a_{3}^{5} \sqrt{3} a_{2}+6 a_{3} a_{2}^{\prime} a_{3}^{\prime} a_{2}^{2}+6 a_{3}^{\prime} a_{2} a_{3}^{3} a_{1}^{\prime}+a_{1} a_{3}^{3} c a_{3}^{\prime} a_{2}-50 a_{3} \sqrt{3} a_{2}^{\prime} a_{3}^{\prime 2} a_{2} \\
& -5 c \sqrt{3} a_{3} a_{3}^{\prime 2} a_{2}^{2}+a_{3}^{\prime} \sqrt{3} a_{2} a_{3}^{4} a_{0}-2 a_{1} \sqrt{3} a_{3}^{5} a_{1}^{\prime}+8 a_{1} \sqrt{3} a_{3}^{2} a_{3}^{\prime 3}+8 a_{1} a_{3}^{3} a_{2}^{\prime} a_{3}^{\prime}-10 a_{1} a_{3}^{2} a_{3}^{\prime 2} a_{2} \\
& -a_{1} \sqrt{3} a_{3}^{3} a_{2}^{\prime} a_{2}-2 a_{1} \sqrt{3} a_{3}^{3} c a_{3}^{\prime 2}+a_{1} \sqrt{3} a_{3}^{2} a_{3}^{\prime} a_{2}^{2}-3 a_{3}^{6} a_{0}^{\prime \prime}-3 a_{3}^{4} a_{2}^{(i v)}-c \sqrt{3} a_{3}^{3} a_{2}^{\prime \prime} a_{2} \\
& +11 a_{3}^{\prime} \sqrt{3} a_{2} a_{3}^{2} a_{2}^{\prime \prime}+16 \sqrt{3} a_{3}^{2} a_{3}^{\prime \prime} a_{2}^{\prime} a_{2}-8 c^{2} \sqrt{3} a_{3}^{3} a_{3}^{\prime} a_{3}^{\prime \prime}+112 c \sqrt{3} a_{3}^{2} a_{3}^{\prime 2} a_{3}^{\prime \prime}+c \sqrt{3} a_{3}^{2} a_{3}^{\prime \prime} a_{2}^{2} \\
& -21 \sqrt{3} a_{3} a_{3}^{\prime \prime} a_{3}^{\prime} a_{2}^{2}-10 a_{1} \sqrt{3} a_{3}^{3} a_{3}^{\prime \prime} a_{3}^{\prime}+2 a_{1} \sqrt{3} a_{3}^{4} c a_{3}^{\prime \prime}+4 \sqrt{3} a_{3}^{4} a_{1}^{\prime \prime} a_{3}^{\prime}=0 .
\end{aligned}
$$

Since this system has infinite solutions we look for some particular solutions. 
3.1.1. $R(u, x)=-\frac{1}{2} x^{2} u^{3}+3 u^{2}+\frac{1}{2} c^{2} u$

If we assume $a_{3}(x)=\sqrt{3} x$, and $a_{2}(x), a_{1}(x), a_{0}(x)$ to be constants then from system (3.11)-(3.12) we obtain that

$$
R(u, x)=-\frac{1}{2} x^{2} u^{3}+3 u^{2}+\frac{1}{2} c^{2} u
$$

and

$$
s s_{1}(t, x)=\frac{3 x}{2}, \quad s s_{2}(t, x)=-\frac{1+c x}{x}, \quad s s_{3}(t, x)=c \frac{-2+3 c x}{4 x}, \quad s s_{4}(t, x)=0 .
$$

Thus, (3.3) becomes

$$
\eta=-\frac{x^{3} u^{3}+2 c u-c^{2} x u+6 x^{2} u G-4 G}{4 x}
$$

namely

$$
u_{x x}=-\frac{x^{3} u^{3}+2 c u-c^{2} x u+6 x^{2} u u_{x}-4 u_{x}}{4 x}
$$

that can be solved in closed form, i.e.

$$
u(t, x)=\frac{c^{2} R_{2}(t) e^{\frac{c x}{2}}-c^{2}(1+c x) e^{\frac{-c x}{2}}}{R_{1}(t)+(c x-2) R_{2}(t) e^{\frac{c x}{2}}+\left(10+5 c x+c^{2} x^{2}\right) e^{\frac{-c x}{2}}},
$$

with $R_{k}(t), k=1,2$ arbitrary functions of $t$. Substituting (3.17) into (1.1) yields the following nonclassical symmetry solution

$$
u(t, x)=\frac{c^{2} c_{1} e^{c^{2} t+\frac{c x}{2}}-c^{2}(1+c x) e^{\frac{-c x}{2}}}{c_{2} e^{\frac{-c^{2} t}{4}}+c_{1}(c x-2) e^{c^{2} t+\frac{c x}{2}}+\left(10+5 c x+c^{2} x^{2}\right) e^{\frac{-c x}{2}}},
$$

with $c_{k}, k=1,2$ arbitrary constants. We observe that

$$
\lim _{t \rightarrow \infty} u(t, x)=\frac{c^{2}}{c x-2}, \quad \lim _{x \rightarrow \pm \infty} u(t, x)=0
$$

and that $u(t, x)<0$ for $t>0, x<0$. This means that the solution (3.18) is not defined at $x=2 / c$ and is positive $\mathrm{g}^{\mathrm{g}}$ if $x \geq 0$.

$\overline{\mathrm{g}}$ It depends also on the values given to the arbitrary constants. 
3.1.2. $R(u, x)=-\frac{1}{2} e^{c x} u^{3}+\frac{c^{2}}{4} u+e^{\frac{c x}{2}}$

If we assume $a_{3}(x)=\sqrt{3} e^{c x}, a_{2}(x)=0$, and $a_{1}(x)=b_{1}, a_{0}(x)=b_{0}$, i.e. constants, then from system (3.11)-(3.12) we obtain that

$$
R(u, x)=-\frac{1}{2} e^{c x} u^{3}+b_{1} u+b_{0} e^{\frac{c x}{2}}, \quad\left[b_{1}, b_{0}=\text { const. }\right]
$$

and thus $\eta$ becomes

$$
\eta=-\frac{1}{8}\left(2 e^{c x} u^{3}+\left(3 c^{2}-4 b_{1}\right) u+8 c G+6 e^{\frac{c x}{2}} c u^{2}+12 e^{\frac{c x}{2}} u G-4 b_{0} e^{-\frac{c x}{2}}\right),
$$

namely

$$
u_{x x}=-\frac{1}{8}\left(2 e^{c x} u^{3}+\left(3 c^{2}-4 b_{1}\right) u+8 c u_{x}+6 e^{\frac{c x}{2}} c u^{2}+12 e^{\frac{c x}{2}} u u_{x}-4 b_{0} e^{-\frac{c x}{2}}\right),
$$

that can be solved in closed form, although the solution is very lengthy. If we assume

$$
b_{1}=\frac{c^{2}}{4}, \quad b_{0}=1
$$

then the solution of (3.22) becomes:

$$
\begin{aligned}
u(t, x)= & \frac{\sqrt[3]{2}}{2\left(R_{1}(t) e^{\frac{\sqrt[3]{2} x}{2}}-R_{2}(t) e^{-\frac{\sqrt[3]{2} x}{4}} \sin \left(\frac{\sqrt[3]{2} \sqrt{3} x}{4}\right)+e^{-\frac{\sqrt[3]{2} x}{4}} \cos \left(\frac{\sqrt[3]{2} \sqrt{3} x}{4}\right)\right) e^{\frac{c x}{2}}} \times \\
& \times\left[2 R_{1}(t) e^{\frac{\sqrt[3]{2} x}{2}}+R_{2}(t) e^{\frac{-\sqrt[3]{2} x}{4}}\left(\sin \left(\frac{\sqrt[3]{2} \sqrt{3} x}{4}\right)-\sqrt{3} \cos \left(\frac{\sqrt[3]{2} \sqrt{3} x}{4}\right)\right)\right. \\
& \left.-e^{\frac{-\sqrt[3]{2} x}{4}}\left(\sqrt{3} \sin \left(\frac{\sqrt[3]{2} \sqrt{3} x}{4}\right)+\cos \left(\frac{\sqrt[3]{2} \sqrt{3} x}{4}\right)\right)\right]
\end{aligned}
$$

which if replaced into (1.1) yields

$$
R_{1}(t)=0, \quad R_{2}(t)=-\tan \left(\frac{3 \sqrt{3} \sqrt[3]{4}}{8}\left(t+c_{1}\right)\right)
$$

This solution oscillates between negative and positive values. Consequently it is not a valid solution for the biological model set in [5]. However, equation

$$
u_{t}=u_{x x}+c u_{x}-\frac{1}{2} e^{c x} u^{3}+\frac{c^{2}}{4} u+e^{\frac{c x}{2}}
$$

maybe of interest for other biological or physical models. 
3.1.3. $R(u, x)=-\frac{u^{3}}{6}-\frac{\sqrt{3} u^{2}}{x}+\frac{c^{2} u}{6}+\frac{\sqrt{3} c^{2}}{3 x}$

If we assume $a_{3}(x)=b_{3}, a_{1}(x)=b_{1}$ and substitute them into system (3.11)-(3.12), after some further simplifications such $b_{1}=c^{2} / 6$ and having to impose that ${ }^{\mathrm{h}} b_{3}=-1$, then we obtain

$$
R(u, x)=-\frac{u^{3}}{6}-\frac{\sqrt{3} u^{2}}{x}+\frac{c^{2} u}{6}+\frac{\sqrt{3} c^{2}}{3 x} .
$$

Thus (3.3) becomes

$$
\eta=\frac{36 x G-36 u+6 \sqrt{3} G u x^{2}-u^{3} x^{2}-6 \sqrt{3} u^{2} x+c^{2} u x^{2}+12 \sqrt{3} c+2 \sqrt{3} c^{2} x}{12 x^{2}}
$$

namely

$$
u_{x x}=\frac{36 x u_{x}-36 u+6 \sqrt{3} u_{x} u x^{2}-u^{3} x^{2}-6 \sqrt{3} u^{2} x+c^{2} u x^{2}+12 \sqrt{3} c+2 \sqrt{3} c^{2} x}{12 x^{2}} .
$$

If we assume $c=0$ then we find that its solution is

$$
u(t, x)=-\frac{2 \sqrt{3}\left(4 R_{2}(t) x^{3}+2 x\right)}{R_{1}(t)+R_{2}(t) x^{4}+x^{2}}
$$

that substituted into (1.1) yields the following solution

$$
u(t, x)=\frac{4 \sqrt{3} x\left(2 x^{2}+c_{1}+12 t\right)}{6 c_{1} t+36 t^{2}-c_{2}-x^{4}-x^{2} c_{1}-12 t x^{2}} .
$$

Although this solution is not valid for the biological problem set in [5] since $c=0$, we are reporting it since equation

$$
u_{t}=u_{x x}+\frac{u^{2}(x u+6 \sqrt{3})}{6 x}
$$

maybe of interest for other biological or physical problems. We observe that solution (3.31) is such that

$$
\lim _{t \rightarrow \infty} u(t, x)=0, \quad \lim _{x \rightarrow \pm \infty} u(t, x)=0
$$

Moreover (3.31) is not defined for the following set of values of $x$ and $t$ :

$$
\begin{array}{r}
\left\{x=\frac{1}{2} \sqrt{-2 c_{1}-24 t+2 \sqrt{c 1^{2}+48 c_{1} t+288 t^{2}-4 c_{2}}}, \forall t\right\}, \\
\left\{x=-\frac{1}{2} \sqrt{-2 c_{1}-24 t+2 \sqrt{c 1^{2}+48 c_{1} t+288 t^{2}-4 c_{2}}}, \forall t\right\}, \\
\left\{x=\frac{1}{2} \sqrt{-2 c_{1}-24 t-2 \sqrt{c 1^{2}+48 c_{1} t+288 t^{2}-4 c_{2}}}, \forall t\right\}, \\
\left\{x=-\frac{1}{2} \sqrt{-2 c_{1}-24 t-2 \sqrt{c 1^{2}+48 c_{1} t+288 t^{2}-4 c_{2}}}, \forall t\right\} .
\end{array}
$$

$\overline{{ }^{\mathrm{h}} \text { It is also possible to have }} b_{3}=1$ although it leads to very lengthy calculations. 
3.1.4. $R(u, x)=-u^{3}+6 \frac{u}{x^{2}}+6 \frac{\sqrt{2}}{x^{3}},[c=0]$

If we impose $a_{3}(x)=b_{3}, a_{2}(x)=0$, and $c=0$, then we obtain

$$
R(u, x)=-u^{3}+6 \frac{u}{x^{2}}+6 \frac{\sqrt{2}}{x^{3}} .
$$

Thus (3.3) becomes

$$
\eta=-\frac{6 \sqrt{2}-6 x u+3 \sqrt{2} x^{3} u G+x^{3} u^{3}}{2 x^{3}}
$$

namely

$$
u_{x x}=-\frac{6 \sqrt{2}-6 x u+3 \sqrt{2} x^{3} u u_{x}+x^{3} u^{3}}{2 x^{3}} .
$$

We find its solution, i.e.

$$
u(t, x)=\frac{\sqrt{2}\left(-R_{1}(t)+3 R_{2}(t) x^{4}+x^{2}\right)}{x\left(R_{1}(t)+R_{2}(t) x^{4}+x^{2}\right)}
$$

that substituted into (1.1) yields the following solution

$$
u(t, x)=-\frac{3 \sqrt{2}\left(12 c_{2}^{2}+24 c_{2} t+4 c_{2} x^{2}+4 c_{1}+12 t^{2}+4 t x^{2}+x^{4}\right)}{x\left(36 c_{2}^{2}+72 c_{2} t-12 c_{2} x^{2}+12 c_{1}+36 t^{2}-12 t x^{2}-x^{4}\right)} .
$$

Although this solution is not valid for the biological problem set in [5] since $c=0$, we report it here because equation

$$
u_{t}=u_{x x}-u^{3}+6 \frac{u}{x^{2}}+6 \frac{\sqrt{2}}{x^{3}}
$$

maybe of interest for other biological or physical problems. We observe that solution (3.42) is such that

$$
\lim _{t \rightarrow \infty} u(t, x)=-\frac{\sqrt{2}}{x}, \quad \lim _{x \rightarrow \pm \infty} u(t, x)=0
$$


and that $u(t, x)<0$ for $t>0, x<0$. Moreover (3.42) is not defined for the following set of values of $x$ and $t$ :

$$
\begin{array}{r}
\left\{x=\sqrt{\left.-6 t-6 c_{2}+2 \sqrt{18 t^{2}+36 t c_{2}+18 c_{2}^{2}+3 c_{1}}, \forall t\right\},},\right. \\
\left\{x=-\sqrt{-6 t-6 c_{2}+2 \sqrt{18 t^{2}+36 t c_{2}+18 c_{2}^{2}+3 c_{1}}, \forall t}\right\}, \\
\left\{x=\sqrt{-6 t-6 c_{2}-2 \sqrt{18 t^{2}+36 t c_{2}+18 c_{2}^{2}+3 c_{1}}}, \forall t\right\}, \\
\left\{x=-\sqrt{\left.-6 t-6 c_{2}-2 \sqrt{18 t^{2}+36 t c_{2}+18 c_{2}^{2}+3 c_{1}}, \forall t\right\},},\right. \\
\left\{\forall x, t=\frac{1}{6} x^{2}-c_{2}+\frac{1}{6} \sqrt{2 x^{4}-12 c_{1}}\right\}, \\
\left\{\forall x, t=\frac{1}{6} x^{2}-c_{2}-\frac{1}{6} \sqrt{2 x^{4}-12 c_{1}}\right\} .
\end{array}
$$

3.2. Case 2. $R(u, x)=\frac{f(u)}{k^{2}(x)}$

If we assume $s s_{1}=0$ then $V_{2}(t, x, u)=s s_{2}(t, x)$ and $d_{0}$ yields that

$$
R(u, x)=\frac{f(u)}{k^{2}(x)}, \quad s s_{2}=k(x) .
$$

Following [11] we impose $F(t, x, u)=0$, thus the annihilation of $d_{1}$ imposes that $k(x)$ is either one the three particular functions of $x$ in (3.9) and their nonclassical symmetry operators are

$$
\partial_{t}-\frac{c x+2}{2 x} \partial_{x} \quad \partial_{t}+\frac{c}{e^{c\left(b_{0}-x\right)}-1} \partial_{x}, \quad \partial_{t}+\left(\frac{1}{b_{1}} \tan \left(\frac{x+b_{2}}{b_{1}}\right)-\frac{c}{2}\right) \partial_{x},
$$

respectively. In each case we can solve the corresponding invariant surface condition (2.3) and reduce the original diffusion equation (1.1) to an ordinary differential equation that involves an arbitrary function of the unknown due to the arbitrariness of $f(u)$. We consider some instances where $f(u)$ has a given expression in order to derive the nonclassical symmetry solution of equation (1.1).

3.2.1. $k(x)=-\frac{c x+2}{2 x}$

We solve the invariant surface equation (2.3), i.e.

$$
u_{t}-\frac{c x+2}{2 x} u_{x}=0
$$

and derive its complete solution as

$$
u(t, x)=H(\xi), \quad \xi=\frac{4 \log (c x+2)-2 c x-c^{2} t}{c^{2}}
$$


where $H(\xi)$ is an arbitrary function of $\xi$. After substituting this solution into equation (1.1), i.e.

$$
u_{t}=u_{x x}+c u_{x}+\frac{4 x^{2}}{(c x+2)^{2}} f(u)
$$

we obtain the following ordinary differential equation

$$
4 \frac{\mathrm{d}^{2} H}{\mathrm{~d} \xi^{2}}-c^{2} \frac{\mathrm{d} H}{\mathrm{~d} \xi}+4 f(H)=0 .
$$

Let us consider $f(u)=1 / u$. In this instance equation (3.57) admits a two-dimensional nonabelian transitive Lie point symmetry algebra ${ }^{\mathrm{i}}$ generated by

$$
\partial_{\xi}, \quad e^{\frac{c^{2} \xi}{4}}\left(4 \partial_{\xi}+c^{2} H \partial_{H}\right),
$$

and equation (3.57) can be integrated by quadrature. In fact taking a canonical representation of the generators of the two-dimensional Lie point symmetry algebra, i.e.

$$
4 e^{\frac{c^{2} \xi}{4}}\left(4 \partial_{\xi}+c^{2} H \partial_{H}\right), \quad-\frac{4}{c^{2}} \partial_{\xi}+4 e^{\frac{c^{2} \xi}{4}}\left(4 \partial_{\xi}+c^{2} H \partial_{H}\right),
$$

we can derive the corresponding canonical variables, i.e.

$$
\tilde{\xi}=H e^{\frac{-c^{2} \xi}{4}}, \quad \tilde{H}=1+e^{\frac{-c^{2} \xi}{4}}\left(-\frac{1}{4 c^{2}}+H\right) .
$$

These variables transform equation (3.57) into its canonical form, i.e.

$$
\frac{\mathrm{d}^{2} \tilde{H}}{\mathrm{~d} \tilde{\xi}^{2}}=\frac{1}{\tilde{\xi}}\left(256\left(\frac{\mathrm{d} \tilde{H}}{\mathrm{~d} \tilde{\xi}}\right)^{3}-3\left(\frac{\mathrm{d} \tilde{H}}{\mathrm{~d} \tilde{\xi}}\right)^{2}+3 \frac{\mathrm{d} \tilde{H}}{\mathrm{~d} \tilde{\xi}}-1\right)
$$

that can be solved by two quadratures and thus its general solution is

$$
\tilde{H}=\tilde{\xi}+c_{2} \pm 16 \int \frac{\mathrm{d} \tilde{\xi}}{\sqrt{2 c_{1}-2 \log (\tilde{\xi})}} .
$$

Unfortunately the last integral cannot be expressed in finite terms.

If we assume $c=0$ then (3.56) becomes

$$
u_{t}=u_{x x}+x^{2} f(u)
$$

In [11] the same nonclassical symmetry was determined if $f(u)=u^{2}(1-u)-(\mathrm{i})$ in(1.3) -. We found that this is true for any $f(u)$.

${ }^{\mathrm{i}}$ We recall that the classification of real two-dimensional Lie symmetry algebra and derivation of corresponding canonical variables were done by Lie himself [35], retold in Bianchi's 1918-textbook [6] and also in more recent textbooks, e.g. [32]. 
3.2.2. $k(x)=\frac{c}{e^{c\left(b_{0}-x\right)}-1}$

We solve the invariant surface equation (2.3), i.e.

$$
u_{t}+\frac{c}{e^{c\left(b_{0}-x\right)}-1} u_{x}=0
$$

and derive its complete solution as

$$
u(t, x)=H(\gamma), \quad \gamma=-\frac{c x+c^{2} t+e^{c\left(b_{0}-x\right)}}{c^{2}},
$$

where $H(\gamma)$ is an arbitrary function of $\gamma$. After substituting this solution into equation (1.1), i.e.

$$
u_{t}=u_{x x}+c u_{x}+\frac{\left(e^{c\left(b_{0}-x\right)}-1\right)^{2}}{c^{2}} f(u),
$$

we obtain the following ordinary differential equation

$$
\frac{\mathrm{d}^{2} H}{\mathrm{~d} \gamma^{2}}+f(H)=0
$$

Its general solution in implicit form is

$$
\pm \int \frac{\mathrm{d} H}{\sqrt{c_{1}-2 \int f(H) \mathrm{d} H}}-\gamma-c_{2}=0 .
$$

Let us consider some instances:

(A) $f(u)=u^{2} \Longrightarrow u(t, x)=-6$ WeierstrassP $\left(\gamma+c_{1}, 0, c_{2}\right)$,

where WeierstrassP represents Weierstrass elliptic function.

(B) $f(u)=u^{3} \Longrightarrow u(t, x)=c_{2} \operatorname{JacobiSN}\left(\left(\frac{\gamma}{\sqrt{2}}+c_{1}\right) c_{2}, \sqrt{-1}\right)$

where $\operatorname{JacobiSN}(z, k)=\sin (\operatorname{JacobiAM}(z, k))$ and JacobiAM represents the Jacobi amplitude function am.

(C) $f(u)=u^{2}(1-u) \Longrightarrow \int \frac{6 \mathrm{~d} H}{\sqrt{18 H^{4}-24 H^{3}+36 c_{1}}}-\gamma-c_{2}=0$

Let us consider two particular values of $c_{1}$.

If we assume $c_{1}=\frac{1}{6}$ then we obtain

$$
u(t, x)=\frac{(1-H) \sqrt{18 H^{2}+12 H+6}}{\sqrt{18 H^{4}-24 H^{3}+6}} \operatorname{arctanh}\left(\frac{2(1+2 H)}{\sqrt{18 H^{2}+12 H+6}}\right)-\gamma-c_{2}=0,
$$

although still an implicit solution of (3.66);

instead $c_{1}=0$ yields

$$
u(t, x)=-\frac{12}{4 c_{2} \gamma+2 c_{2}^{2}+2 \gamma^{2}-9}
$$


a nonclassical symmetry solution of equation (3.66), i.e.

$$
u_{t}=u_{x x}+c u_{x}+\frac{\left(e^{c\left(b_{0}-x\right)}-1\right)^{2}}{c^{2}} u^{2}(1-u)
$$

This solution tends to zero when $t$ (or $x$ ) goes to infinity. Also it blows up in finite time if

$$
t=\frac{1}{2 c^{2}}\left(2 c_{2} c^{2}-2 c x-2 e^{c\left(b_{0}-x\right)} \pm c^{2} \sqrt{18}\right), \quad \forall x .
$$

3.2.3. $k(x)=\frac{1}{b_{1}} \tan \left(\frac{x+b_{2}}{b_{1}}\right)-\frac{c}{2}$

We solve the invariant surface equation (2.3), i.e.

$$
u_{t}+\left(\frac{1}{b_{1}} \tan \left(\frac{x+b_{2}}{b_{1}}\right)-\frac{c}{2}\right) u_{x}=0
$$

and derive its complete solution as

$$
\begin{aligned}
u(t, x)= & H(\rho), \\
\rho= & t+\frac{2 b_{1}}{4+b_{1}^{2} c^{2}}\left[c\left(x+b_{2}\right)+\log \left(1+\tan ^{2}\left(\frac{x+b_{2}}{b_{1}}\right)\right)\right. \\
& \left.-2 \log \left(2 \tan \left(\frac{x+b_{2}}{b_{1}}\right)-b_{1} c\right)\right],
\end{aligned}
$$

where $H(\rho)$ is an arbitrary function of $\rho$. After substituting this solution into equation (1.1), i.e.

$$
u_{t}=u_{x x}+c u_{x}+\frac{4 b_{1}^{2}}{\left(2 \tan \left(\frac{x+b_{2}}{b_{1}}\right)-b_{1} c\right)^{2}} f(u),
$$

we obtain the following ordinary differential equation

$$
4 b_{1}^{2} \frac{\mathrm{d}^{2} H}{\mathrm{~d} \rho^{2}}+\left(4+b_{1}^{2} c^{2}\right) \frac{\mathrm{d} H}{\mathrm{~d} \rho}+4 b_{1}^{2} f(H)=0 .
$$

If $f(u)=u$, namely if equation (3.72) is linear then we obtain that the general solution is

$$
\begin{gathered}
u(t, x)=c_{1} e^{-\frac{4+b_{1}^{2} c^{2}-\rho \sqrt{\left(b_{1}^{2} c^{2}+4\right)^{2}-64 b_{1}^{4}}}{8 b_{1}^{2}}} \\
+c_{2} e^{-\frac{4+b_{1}^{2} c^{2}+\rho \sqrt{\left(b_{1}^{2} c^{2}+4\right)^{2}-64 b_{1}^{4}}}{8 b_{1}^{2}}} .
\end{gathered}
$$

\section{Final remarks}

The application of the nonclassical symmetry method to equation (1.1) yields different possible expressions of $R(u, x)$ and in several instances even a class of solutions in finite form. These solutions may not be all suitable for the problem set in [5] since they may take negative values, and also have singularities for finite values of $t$ and $x$. 
However, we have found that nonclassical symmetries exist for the following reaction-diffusion equations, i.e.

$$
u_{t}=u_{x x}+c u_{x}-\frac{a_{3}^{2}(x)}{6} u^{3}+\frac{a_{2}(x)}{2} u^{2}+a_{1}(x) u+a_{0}(x),
$$

with $a_{0}, a_{1}, a_{2}, a_{3}$ satisfying system (3.11)-(3.12). Particular instances have been obtained, i.e.

$$
\begin{array}{r}
u_{t}=u_{x x}+c u_{x}-\frac{1}{2} x^{2} u^{3}+3 u^{2}+\frac{1}{2} c^{2} u, \\
u_{t}=u_{x x}+c u_{x}-\frac{1}{2} e^{c x} u^{3}+\frac{c^{2}}{4} u+e^{\frac{c x}{2}} \\
u_{t}=u_{x x}+c u_{x}-\frac{u^{3}}{6}-\frac{\sqrt{3} u^{2}}{x}+\frac{c^{2} u}{6}+\frac{\sqrt{3} c^{2}}{3 x}, \\
u_{t}=u_{x x}-u^{3}+\frac{6 u}{x^{2}}+\frac{6 \sqrt{2}}{x^{3}} .
\end{array}
$$

Moreover we have found that nonclassical symmetries exist for the following three families in the class of equation (1.1), i.e.

$$
\begin{array}{rr}
u_{t}=u_{x x}+c u_{x}+\frac{4 x^{2}}{(c x+2)^{2}} f(u), & \forall f(u) \\
u_{t}=u_{x x}+c u_{x}+\frac{\left(e^{c\left(b_{0}-x\right)}-1\right)^{2}}{c^{2}} f(u), & \forall f(u) \\
u_{t}=u_{x x}+c u_{x}+\frac{4 b_{1}^{2}}{\left(2 \tan \left(\frac{x+b_{2}}{b_{1}}\right)-b_{1} c\right)^{2}} f(u), & \forall f(u) .
\end{array}
$$

We conclude by observing that the method of heir-equations [46] very much facilitates the search for nonclassical symmetries.

\section{References}

[1] F. Allassia and M.C. Nucci, Symmetries and heir equations for the laminar boundary layer model, $J$. Math. An. Appl. 201 (1996) 911-942.

[2] D.J. Arrigo, P. Broadbridge, J.M. Hill, Nonclassical symmetry solutions and the methods of BlumanCole and Clarkson-Kruskal, J. Math. Phys. 34 (1993) 4692-4703.

[3] D.J. Arrigo, P. Broadbridge, J.M. Hill, Nonclassical symmetry reductions of the linear diffusion equation with a nonlinear source, IMA J. Appl. Math. 52 (1994) 1-24.

[4] D.J. Arrigo, D.A. Ekrut, J.R. Fliss, Long Le, Nonclassical symmetries of a class of Burgers systems, $J$. Math. Anal. Appl. 371 (2010) 813-820.

[5] H. Berestycki, O. Diekmann, C.J. Nagelkerke, P.A. Zegeling, Can a Species Keep Pace with a Shifting Climate?, Bull. Math. Biol. 71 (2009) 399-429.

[6] L. Bianchi, Lezioni sulla teoria dei gruppi continui finiti di trasformazioni, Enrico Spoerri, Pisa (1918)

[7] N. Bîlă and J. Niesen, On a new procedure for finding nonclassical symmetries, J. Symbol. Comp. 38 (2004) 1523-1533.

[8] G.W. Bluman and J.D. Cole, The general similarity solution of the heat equation, J. Math. Mech. 18 (1969) 1025-1042.

[9] G.W. Bluman, G.J. Reid, S. Kumei, New classes of symmetries for partial differential equations, $J$. Math. Phys. 29 (1988) 806-811. 
[10] G.W. Bluman, S.-f. Tian, Z. Yang, Nonclassical analysis of the nonlinear Kompaneets equation J. Eng. Math. (2012).

[11] B.H. Bradshaw-Hajek, M.P. Edwards, P. Broadbridge, G.H. Williams, Nonclassical symmetry solutions for reactiondiffusion equations with explicit spatial dependence, Nonlin. Anal. 67 (2007) 2541-2552.

[12] M.S. Bruzón and M.L. Gandarias, Applying a new algorithm to derive nonclassical symmetries, Commun. Nonlinear Sci. Numer. Simul. 13 (2008) 517-523.

[13] R. Cherniha, New Q-conditional symmetries and exact solutions of some reaction-diffusion-convection equations arising in mathematical biology, J. Math. Anal. Appl. 326 (2007) 783-799.

[14] R. Cherniha and V. Davydovych, Conditional symmetries and exact solutions of nonlinear reactiondiffusion systems with non-constant diffusivities, Commun. Nonlinear Sci. Numer. Simulat. 17 (2012) 3177-3188.

[15] R. Cherniha and O. Pliukhin, New conditional symmetries and exact solutions of reaction-systems with power diffusivities, J. Phys. A: Math. Gen. 41 (2008) 185208-185222.

[16] R.M. Cherniha and M.I. Serov, Symmetries, Ansätze and exact solutions of nonlinear second-order evolution equations with convection term, European J. Appl. Math. 9 (1998) 527-542.

[17] R. Cherniha and M. Serov, Nonlinear systems of the Burgers-type equations: Lie and Q-conditional symmetries, Ansätze and solutions, J. Math. Anal. Appl. 282 (2003) 305-328.

[18] P.A. Clarkson, Nonclassical symmetry reductions of nonlinear partial differential equations, Math. Comput. Modell. 18 (1993) 45-68.

[19] P.A. Clarkson and E.L. Mansfield, Symmetry reductions and exact solutions of a class of nonlinear heat equations, Physica D 70 (1993) 250-288.

[20] P.A. Clarkson and P. Winternitz, Nonclassical symmetry reductions for the Kadomtsev-Petviashvili equation, Physica D 49 (1991) 257-272.

[21] V.G. Dulov, S.P. Novikov, L.V. Ovsyannikov, B.L. Rozhdestvenskii, A.A. Samarskii, Yu.I. Shokin, Nikolai Nikolaevich Yanenko (obituary), Russ. Math. Surv. 39 (1984) 99-110.

[22] P.G. Estevez, P.R. Gordoa, Nonclassical symmetries and the singular manifold method: The Burgers equation, Theor. Math. Phys. 99 (1994) 562-566

[23] A.S. Fokas and Q.M. Liu, Generalised conditional symmetries and exact solutions of nonintegrable equations, Theor. Math. Phys. 99 (1994) 263-277.

[24] W.I. Fushchych, M.I. Serov, L.A. Tulupova, The conditional invariance and exact solutions of the nonlinear diffusion equation, Proc. Acad. of Sci. Ukraine 4 (1993) 37-40.

[25] W.I. Fushchych, W.M. Shtelen, M.I. Serov, Symmetry Analysis and Exact Solutions of Equations of Nonlinear Mathematical Physics, Kluwer, Dordrecht, (1993).

[26] W.I. Fushchych, W.M. Shtelen, M.I. Serov, R.O. Popovych, Q-conditional symmetry of the linear heat equation, Proc. Acad. of Sci. Ukraine 12 (1992) 28-33.

[27] M.L. Gandarias, Nonclassical symmetries of a porous medium equation with absorption, J. Phys. A: Math. Gen. 30 (1997) 6081-6091.

[28] M.L. Gandarias, J.L. Romero, J.M. Díaz, Nonclassical symmetry reductions of a porous medium equation with convection, J. Phys. A: Math. Gen. 32 (1999) 1461-1473.

[29] J. Goard, Generalised conditional symmetries and Nucci's method of iterating the nonclassical symmetries method, Appl. Math. Lett. 16 (2003) 481-486.

[30] G. Guthrie, Constructing Miura transformations using symmetry groups, Research Report No. 85 (1993).

[31] D.-J. Huang and S. Zhou, Group-theoretical analysis of variable coefficient nonlinear telegraph equations, Acta Appl. Math. 117 (2012) 135-183.

[32] N.H. Ibragimov, Elementary Lie Group Analysis and Ordinary Differential Equations, Wiley, Chichester (1999).

[33] B. Kruglikov, Symmetry approaches for reductions of PDEs, differential constraints and LagrangeCharpit method, Acta Appl. Math. 101 (2008) 145-161.

[34] D. Levi and P. Winternitz, Nonclassical symmetry reduction: Example of the Boussinesq equation, $J$. Phys. A: Math. Gen. 22 (1989) 2915-2924. 
[35] S. Lie, Vorlesungen über Differentialgleichungen mit bekannten infinitesimalen Transformationen, Teubner, Leipzig (1912).

[36] S.-Y. Lou, Nonclassical symmetry reductions for the dispersive wave equations in shallow water, $J$. Math. Phys. 33 (1992) 4300-4305.

[37] D.K. Ludlow, P.A. Clarkson, A.P. Bassom, Nonclassical symmetry reductions of the three-dimensional incompressible Navier-Stokes equations, J. Phys. A: Math. Gen. 31 (1998) 7965-7980.

[38] S. Martini, N. Ciccoli, M.C. Nucci, Group analysis and heir-equations of a mathematical model for thin liquid films, J. Nonlinear Math. Phys. 16 (2009) 77-92.

[39] S.V. Meleshko, Methods for Constructing Exact Solutions of Partial Differential Equations: Mathematical and Analytical Techniques with Applications to Engineering, Springer, New York (2005)

[40] M.C. Nucci, Interactive REDUCE programs for calculating classical, non-classical, and approximate symmetries of differential equations, in Computational and Applied Mathematics II. Differential Equations, W.F. Ames and P.J. Van der Houwen, Eds., Elsevier, Amsterdam (1992) 345-350.

[41] M.C. Nucci, Symmetries of linear, C-integrable, S-integrable, and non-integrable equations, in Nonlinear Evolution Equations and Dynamical Systems. Proceedings NEEDS '91, M. Boiti, L. Martina, and F. Pempinelli, Eds., World Scientific, Singapore (1992) 374-381.

[42] M.C. Nucci, Nonclassical symmetries and Bäcklund transformations, J. Math. An. Appl. 178 (1993) 294-300.

[43] M.C. Nucci, Iterating the nonclassical symmetries method, Physica D 78 (1994) 124-134.

[44] M.C. Nucci, Iterations of the nonclassical symmetries method and conditional Lie-Bäcklund symmetries, J. Phys. A: Math. Gen. 29 (1996) 8117-8122.

[45] M.C. Nucci, Interactive REDUCE programs for calculating Lie point, non-classical, Lie-Backlund, and approximate symmetries of differential equations: manual and floppy disk, in CRC Handbook of Lie Group Analysis of Differential Equations. Vol. 3: New Trends in Theoretical Developments and Computational Methods, ed. N. H. Ibragimov, CRC Press, Boca Raton (1996) 415-481.

[46] M.C. Nucci, Nonclassical symmetries as special solutions of heir-equations, J. Math. Anal. Appl. 279 (2003) 168-179.

[47] M.C. Nucci and W.F. Ames, Classical and nonclassical symmetries of the Helmholtz equation, J. Math. Anal. Appl. 178 (1993) 584-591.

[48] M.C. Nucci and P.A. Clarkson, The nonclassical method is more general than the direct method for symmetry reduction. An example of the Fitzhugh-Nagumo equation, Phys. Lett. A 184 (1992) 49-56.

[49] P.J. Olver, Direct reduction and differential constraints, Proc. R. Soc. Lond. A 444 (1994) 509-523.

[50] A. Oron and P. Rosenau, Some symmetries of the nonlinear heat and wave equations, Phys. Lett. A 118 (1986) 172-176.

[51] R.O. Popovych, Reduction operators of linear second-order parabolic equations, J. Phys. A: Math. Theor. 41 (2008) 185202 (31pp)

[52] E. Pucci, Similarity reductions of partial differential equations, J. Phys. A: Math. Gen. 25 (1992) 26312640.

[53] A.F. Sidorov, V.P. Shapeev, N.N. Yanenko, The method of differential constraints and its applications in gas dynamics, (in Russian) Nauka, Novosibirsk (1984).

[54] C. Sophocleous, Transformation properties of a variable-coefficient Burgers equation, Chaos, Solitons and Fractals 20 (2004) 1047-1057.

[55] O.O. Vaneeva, R.O. Popovych, C. Sophocleous, Extended group analysis of variable coefficient reactiondiffusion equations with exponential nonlinearities, J. Math. Anal. Appl. 396 (2012) 225-242.

[56] E.M. Vorob'ev, Partial symmetries and integrable multidimensional differential equations, Differ. Eqs. 25 (1989) 322-325.

[57] N.N. Yanenko, Compatibility theory and integration methods for systems of nonlinear partial differential equations, in Proc. Fourth All-Union Math. Congr. in Leningrad 1961, Vol. II (in Russian) Nauka, Leningrad (1964) 247-252.

[58] R.Z. Zhdanov, Conditional Lie-Bäcklund symmetry and reduction of evolution equations, J. Phys. A: Math. Gen. 28 (1995) 3841-3850. 